\title{
THE OTHER CLOSURE AND COMPLETE SUBLOCALES
}

\author{
MARIA MANUEL CLEMENTINO, JORGE PICADO, AND ALEŠ PULTR \\ Dedicated with thanks to our friend Bob Lowen
}

\begin{abstract}
Sublocales of a locale (frame, generalized space) can be equivalently represented by frame congruences. In this paper we discuss, a.o., the sublocales corresponding to complete congruences, that is, to frame congruences which are closed under arbitrary meets, and present a "geometric" condition for a sublocale to be complete. To this end we make use of a certain closure operator on the coframe of sublocales that allows not only to formulate the condition but also to analyze certain weak separation properties akin to subfitness or $T_{1}$.

Trivially, every open sublocale is complete. We specify a very wide class of frames, containing all the subfit ones, where there are no others. In consequence, e.g., in this class of frames, complete homomorphisms are automatically Heyting.
\end{abstract}

\section{INTRODUCTION}

Sublocales $S \subseteq L$ of a frame (locale) are, up to isomorphism, precisely the frames (locales) $S$ embedded into $L$ by extremal monomorphisms in the category of locales. Thus, if we view a frame as a generalized space we have here the natural geometric concept of a generalized subspace. Unlike classical subspaces of a topological space, they have also an algebraic aspect, being in a natural one-one correspondence with the congruences on $L$. Now, among frame congruences on $L$, that is, the congruences closed in $L \times L$ under arbitrary joins and finite meets, we have the special case of complete lattice congruences (closed under all joins and all meets). The question naturally arises what are the geometric features of complete sublocales, that is, of sublocales corresponding to complete congruences.

Date: March 8, 2018.

2010 Mathematics Subject Classification. 06D22, 54B05, 54D10.

Key words and phrases. Frame, locale, frame congruence, sublocale, subfit frame, c-subfit frame, fit frame, regular frame, fitted sublocale, codense sublocale, complete sublocale, weakly complete sublocale. 
In particular, each open sublocale is complete. The case of finite frames immediately shows that there are complete sublocales that are not open. On the other hand, under not too demanding separation axioms, complete frame homomorphisms coincide with the open (Heyting) ones and hence one can expect that under such circumstances also complete and open sublocales will coincide. Thus the questions naturally arise

- what is the geometric relation between complete and open sublocales in general,

- in view of such a relation, what makes the two concepts coincide in special frames,

- and what is the weakest condition under which they still coincide.

It turns out that the key is a closure operator $S \mapsto S^{\circ}$, which we call fitting (following the Isbell terminology of fitted sublocales [?]). We will answer the questions above by presenting a formula in terms of that operator ( $S$ is complete iff for each open $U,(S \cap U)^{\circ}$ is open; assuming just the openness of $S^{\circ}$ also makes a special sense), explain the coincidence under special conditions by the behaviour of this operator, and specify the conditions in these terms.

The paper is organized as follows. After necessary preliminaries in Section 1 we discuss in Section 2 the operator of fitting. An analogous construction in spaces is not a restriction of the general pointfree one; the differences are analyzed in Section 3. Section 4 is devoted to the behavior of completeness (and of its weaker variant) in terms of the fitting operator, and to showing what happens in the context of the separation axiom of subfitness. The last section is concerned with the "border of the coincidence" of completeness and openness. A necessary and sufficient condition for the coincidence in the form of a relaxed subfitness (c-subfitness) is found, and it is also shown that this is precisely the condition under which complete homomorphisms automatically preserve the Heyting operation. It should be stated, though, that while c-subfitness is weaker than subfitness formally, the question whether it is really weaker remains an open problem.

\section{Preliminaries}

1.1. The terminology and notation concerning posets (here they will be mostly complete lattices) is standard. For $A \subseteq(X, \leq)$ we write

$$
\uparrow A=\{x \in X \mid x \geq a \text { for some } a \in A\}
$$


and

$$
\downarrow A=\{x \in X \mid x \leq a \text { for some } a \in A\} .
$$

A join (supremum) of $A \subseteq(X, \leq)$ will be denoted by $\bigvee A$, and we write $a \vee b$ for $\bigvee\{a, b\}$; similarly we write $\bigwedge A$ and $a \wedge b$ for infima.

The smallest resp. largest element in a poset will be denoted by 0 resp. 1 .

1.2. Adjoint maps. If $X, Y$ are posets we say that monotone maps $f: X \rightarrow Y, g: Y \rightarrow X$ are adjoint, $f$ to the left and $g$ to the right, and write $f \dashv g$, if

$$
f(x) \leq y \quad \text { iff } \quad x \leq g(y)
$$

Recall that this is characterized by $f g(y) \leq y$ and $x \leq g f(x)$, that if $f \dashv g$ then $f$ (resp. $g$ ) preserves all the existing suprema (resp. infima), and

1.2.1. if $X, Y$ are complete lattices then an $f: X \rightarrow Y$ preserving all suprema (resp. a $g: Y \rightarrow X$ preserving all infima) has a right (resp. left) adjoint.

1.3. Frames and coframes. A frame, resp. coframe, is a complete lattice $L$ satisfying the distributivity law

$$
\begin{aligned}
(\bigvee A) \wedge b & =\bigvee\{a \wedge b \mid a \in A\}, \\
\operatorname{resp} . \quad(\bigwedge A) \vee b & =\bigwedge\{a \vee b \mid a \in A\},
\end{aligned}
$$

for all $A \subseteq L$ and $b \in L$; a frame (resp. coframe) homomorphism preserves all joins and all finite meets (resp. all meets and all finite joins). The lattice $\Omega(X)$ of all open subsets of a topological space $X$ is an example of a frame, and if $f: X \rightarrow Y$ is continuous we obtain a frame homomorphism $\Omega(f): \Omega(Y) \rightarrow \Omega(X)$ by setting $\Omega(f)(U)=$ $f^{-1}[U]$. Thus we have a functor $\Omega$ from the category of topological spaces into that of frames.

1.4. The Heyting structure. The equality (frm) states that the mappings $(x \mapsto x \wedge b): L \rightarrow L$ preserve all joins. Hence, by ??, every frame is a Heyting algebra with the Heyting operation $\rightarrow$ satisfying

$$
a \wedge b \leq c \quad \text { iff } \quad a \leq b \rightarrow c .
$$

1.4.1. Pseudocomplements, supplements and complements. In a frame we have the pseudocomplement $b^{*}=b \rightarrow 0(=\bigvee\{x \mid x \wedge b=0\})$; dually, in a coframe we have the supplement $b^{\#}=\bigwedge\{x \mid x \vee b=1\}$ $([?])$. Recall the standard De Morgan formulas $\left(\bigvee a_{i}\right)^{*}=\bigwedge a_{i}^{*}$ and $\left(\bigwedge a_{i}\right)^{\#}=\bigvee a_{i}^{\#}$.

An element $a$ is said to be complemented if there exists a $b$ such that $a \wedge b=0$ and $a \vee b=1$; such $b$ will be referred to as the complement 
of $a$. In a distributive lattice the complement, if it exists, is uniquely determined, and is simultaneously the pseudocomplement and the supplement of $a$. Therefore we will also denote it by $a^{*}$ (it will be always clear we have in mind a complement which in the case in question happens to exist).

1.5. The concrete category Loc. The functor $\Omega$ : Top $\rightarrow$ Frm from ?? is a contravariant full embedding on an important part of Top, the subcategory of sober spaces. Thus we can regard frames as a natural generalization of spaces; it is useful to view it as a covariant functor into the category of locales Loc, the dual of the category of frames. Furthermore, it is of advantage to represent Loc as a concrete category with specific maps as morphisms. For this purpose one defines a localic map $f: L \rightarrow M$ as the right adjoint of a frame homomorphism $h=f^{*}: M \rightarrow L$. This can be done since frame homomorphisms preserve suprema; but, of course, not every mapping preserving infima is a localic one: they are precisely the infima-preserving mappings $f: L \rightarrow M$ that satisfy $f\left(f^{*}(b) \rightarrow a\right)=b \rightarrow f(a)$ and $(f(a)=1 \Rightarrow a=1)$ for every $a \in L$ and $b \in M$.

1.6. The coframe of sublocales. A sublocale of a frame $L$ is a subset $S \subseteq L$ such that

(1) $M \subseteq S$ implies $\bigwedge M \in S$, and

(2) if $a \in L$ and $s \in S$ then $a \rightarrow s \in S$.

Note that sublocales are the natural subobjects in the category Loc: extremal monomorphisms in Loc are up to isomorphism precisely the embeddings of sublocales.

The set of all sublocales ordered by inclusion, denoted by $\mathcal{S}(L)$, is a coframe, with lattice operations

$$
\bigwedge_{i \in J} S_{i}=\bigcap_{i \in J} S_{i} \text { and } \bigvee_{i \in J} S_{i}=\left\{\bigwedge A \mid A \subseteq \bigcup_{i \in J} S_{i}\right\}
$$

The top of $\mathcal{S}(L)$ is $L$ and the bottom is the set $\mathrm{O}=\{1\}$ (the empty sublocale).

We have the closed resp. open sublocales

$$
\mathfrak{c}(a)=\uparrow a \quad \text { resp. } \quad \mathfrak{o}(a)=\{x \mid a \rightarrow x=x\}=\{a \rightarrow x \mid x \in L\}
$$

modeling closed resp. open subspaces (and corresponding precisely to the closed resp. open parts in [?]). They are complements of each other, and we have (see e.g. [?]):

(a) $\mathfrak{o}(0)=\mathrm{O}, \mathfrak{o}(1)=L, \mathfrak{o}(a) \cap \mathfrak{o}(b)=\mathfrak{o}(a \wedge b), \bigvee \mathfrak{o}\left(a_{i}\right)=\mathfrak{o}\left(\bigvee a_{i}\right)$,

(b) $\mathfrak{c}(0)=L, \mathfrak{c}(1)=0, \mathfrak{c}(a) \vee \mathfrak{c}(b)=\mathfrak{c}(a \wedge b), \bigcap \mathfrak{c}\left(a_{i}\right)=\mathfrak{c}\left(\bigvee a_{i}\right)$ 
(c) The closed (resp. open) sublocales of a sublocale $S$ are precisely the $\mathfrak{c}(a) \cap S($ resp. $\mathfrak{o}(a) \cap S)$ for $a \in S$.

1.6.1. Partial frame distributivity in $\mathcal{S}(L)$. In $\mathcal{S}(L)$ we have the distributivity rule (cofrm) from ??, and (frm) does not generally hold. But, maybe somewhat surprisingly,

if $\left(T_{i}\right)_{i \in J}$ is any system of sublocales and if $S$ is complemented then

$$
\left(\bigvee_{i \in J} T_{i}\right) \cap S=\bigvee_{i \in J}\left(T_{i} \cap S\right)
$$

(see e.g. [?, VI.4.4.3]).

1.6.2. Frame congruences. Another representation of subobjects is as (natural equivalence classes of) surjective frame homomorphisms, the left adjoints to the embeddings of sublocales. Or, we can take the associated frame congruences $E \subseteq L \times L$ (the sublocale associated with $E$ can be given by the formula $\left.S_{E}=\{\bigvee E a \mid a \in L\}\right)$. The natural correspondence of sublocales and frame homomorphisms gives rise to a contravariant isomorphism between $\mathcal{S}(L)$ and the frame of congruences.

Recall the following fact from [?, VI.1.4.1]:

Theorem. Let $S$ be a sublocale of $L$. Then, for the corresponding congruence $E_{S}$, we have

$$
E_{S}=\{(a, b) \mid \uparrow a \cap S=\uparrow b \cap S\}=\{(a, b) \mid \mathfrak{o}(a) \cap S=\mathfrak{o}(b) \cap S\} .
$$

(For the equivalence of the two formulas see also the proof in [?].)

The frame congruences associated with the open resp. closed sublocales are

$$
\Delta_{a}=\{(x, y) \mid x \wedge a=y \wedge a\} \quad \text { resp. } \quad \nabla_{a}=\{(x, y) \mid x \vee a=y \vee a\} ;
$$

One speaks of open resp. closed congruences.

1.7. Quotients of frames. Let $R \subseteq L \times L$ be an arbitrary relation. The quotient by the congruence generated by $R$ can be constructed as follows (see e.g. [?, III.11]). An element $s \in L$ is $R$-saturated if

$$
\forall a, b, c \quad a R b \Rightarrow a \wedge c \leq s \text { iff } b \wedge c \leq s
$$

(if $R$ is closed under finite meets, in particular if it is already a congruence, the condition simplifies to $a R b \Rightarrow a \leq s$ iff $b \leq s$ ). It is easy to see that the set of $R$-saturated elements is a sublocale of $L$ (usually denoted by $L / R$ ). We have a map $\nu_{R}: L \rightarrow L$ called the nucleus of $R$ (or of the resulting sublocale) which restricts to a quotient frame homomorphism $L \rightarrow L / R$. 
1.8. Subfit, fit and regular. A frame is subfit (conjunctive in [?]) if

$$
\forall a, b, a \not \leq b \Rightarrow \exists c, a \vee c=1 \neq b \vee c,
$$

and fit if

$$
\forall a, b, \quad a \not \leq b \Rightarrow \exists c, a \vee c=1 \text { and } c \rightarrow b \neq b .
$$

A frame is subfit iff each open sublocale is a join of closed ones, and fit iff each closed sublocale is a meet of open ones (those were the original definitions in [?]). A frame is fit iff each of its sublocales is subfit.

In spaces, subfitness is slightly weaker than $T_{1}$ (together with $T_{D}$ see [?] — it is precisely $T_{1}$; for more about low separation axioms see e.g. $[?, ?])$. The fitness, however, is, surprisingly, a strong separation property akin to regularity (see [?]).

Regularity: Write $x \prec y$ if $x^{*} \vee y=1$. A frame $L$ is regular if, for each $a \in L, \quad a=\bigvee\{x \mid x \prec a\}$, and this corresponds precisely to the regularity of spaces.

For more about frames see, e.g., [?] or [?]. We use only the most standard facts about categories; the reader may consult, e.g., [?].

\section{Fitting: the other Closure}

2.1. A sublocale $S$ is fitted (Isbell, [?]) if it is a meet of open ones. The fitted sublocales of a locale $L$ form a closure system in $\mathcal{S}(L)$ (that is, a system of sublocales closed under arbitrary intersections), with associated closure operator

$$
S \mapsto S^{\circ}=\bigcap\{T \mid T \text { fitted, } S \subseteq T\}=\bigcap\{\mathfrak{o}(a) \mid S \subseteq \mathfrak{o}(a)\} .
$$

We call $S^{\circ}$ the fitting of $S$. In particular, we have

$$
S \subseteq S^{\circ}, S \subseteq T \Rightarrow S^{\circ} \subseteq T^{\circ},\left(S^{\circ}\right)^{\circ}=S^{\circ} \text { and } 0^{\circ}=0 .
$$

Furthermore, the coframe distributivity in $\mathcal{S}(L)$ implies immediately that the closure system of fitted sublocales is also closed under binary joins. Hence, the fitting closure also satisfies the property

$$
(S \vee T)^{\circ}=S^{\circ} \vee T^{\circ} \text {. }
$$

Note. We have not been able to find an effective use of this closure operator in the literature on pointfree topology. A. Simpson mentions it implicitly in [?, Section 4], in the framework of $\sigma$-locales, and, more recently, T. Dube [?, Remark 3.3] used a variant of it (defined only for cozero elements $a \in L)$ to characterize $G_{\delta}$-dense sublocales.

Note that the congruences $E$ on $L$ which correspond to the fitted sublocales of $L$ are exactly those for which $E=\bigvee\left\{\Delta_{a} \mid \Delta_{a} \subseteq E\right\}$ in the congruence frame of $L$, that is, the $E$ generated by $\{(a, 1) \mid(a, 1) \in E\}$. 
2.2. Observation. The supplements of fitted sublocales are, obviously, the joins of closed elements:

$$
S^{\#}=(\bigcap\{\mathfrak{o}(a) \mid S \subseteq \mathfrak{o}(a)\})^{\#}=\bigvee\{\mathfrak{c}(a) \mid S \cap \mathfrak{c}(a)=0\}
$$

2.3. In the notation of this section we have (see [?] or [?, Prop. 4.2]) that

- a frame $L$ is fit iff for all $a \in L, \mathfrak{c}(a)^{\circ}=\mathfrak{c}(a)$, and, equivalently,

- a frame $L$ is fit iff for all sublocales $S \subseteq L, S^{\circ}=S$, that is, if fitting is trivial.

2.4. Images and preimages. ([?]) For a localic map $f: L \rightarrow M$ the image $f[S]$ of any sublocale $S \subseteq L$ is a sublocale of $M$. On the other hand, the set-theoretic preimage $f^{-1}[S]$ of a sublocale $S$ is not necessarily a sublocale. It is a meet-closed subset, though, and hence (see the formula for the join of sublocales in ??) there is the largest sublocale $f_{-1}[S]=\bigvee\left\{T \in \mathcal{S}(L) \mid T \subseteq f^{-1}[S]\right\}$ contained in $f^{-1}[S]$. This defines the localic preimage function $f_{-1}: \mathcal{S}(M) \rightarrow \mathcal{S}(L)$ right adjoint to the image. For closed sublocales we have $f_{-1}[\mathfrak{c}(a)]=f^{-1}[\mathfrak{c}(a)]=\mathfrak{c}\left(f^{*}(a)\right)$. For open sublocales the localic and set-theoretic preimages do not necessarily coincide, but we do have $f_{-1}[\mathfrak{o}(a)]=\mathfrak{o}\left(f^{*}(a)\right)$.

Proposition. Let $f: L \rightarrow M$ be a localic map, $S \in \mathcal{S}(L)$ and $T \in$ $\mathcal{S}(M)$. Then:

(1) $f\left[S^{\circ}\right] \subseteq f[S]^{\circ}$.

(2) $f_{-1}[T]^{\circ} \subseteq f_{-1}\left[T^{\circ}\right]$

(3) If $f$ is injective, then $f_{-1}\left[f[S]^{\circ}\right]=S^{\circ}$.

Proof. (1) First, notice that one of the defining properties of a localic map means that $f\left[\mathfrak{o}\left(f^{*}(b)\right)\right] \subseteq \mathfrak{o}(b)$ for any $b \in M$. Then, we have

$$
\begin{aligned}
f\left[S^{\circ}\right] & \subseteq \bigcap\{f[\mathfrak{o}(a)] \mid S \subseteq \mathfrak{o}(a)\} \subseteq \bigcap\left\{f\left[\mathfrak{o}\left(f^{*}(b)\right)\right] \mid S \subseteq \mathfrak{o}\left(f^{*}(b)\right)\right\}= \\
& =\bigcap\left\{f\left[\mathfrak{o}\left(f^{*}(b)\right)\right] \mid f[S] \subseteq \mathfrak{o}(b)\right\} \subseteq \bigcap\{\mathfrak{o}(b) \mid f[S] \subseteq \mathfrak{o}(b)\}=f[S]^{\circ} .
\end{aligned}
$$

(2) It is equivalent to (1) by adjunction.

(3) By the previous property, one has always

$$
S^{\circ} \subseteq f_{-1}[f[S]]^{\circ} \subseteq f_{-1}\left[f[S]^{\circ}\right]
$$

Moreover,

$$
f_{-1}\left[f[S]^{\circ}\right]=\bigcap\left\{\mathfrak{o}\left(f^{*}(b)\right) \mid f[S] \subseteq \mathfrak{o}(b)\right\}=\bigcap\left\{\mathfrak{o}\left(f^{*}(b)\right) \mid S \subseteq \mathfrak{o}\left(f^{*}(b)\right)\right\}
$$

and this is $S^{\circ}$ whenever $f^{*}$ is onto, that is, whenever $f$ is one-one.

Note that property (1) together with ?? make the fitting operator a closure operator in the sense of Dikranjan-Giuli [?]. 
2.5. Codense sublocales. Let $S \subseteq T$ be sublocales of $L$. We say that $S$ is codense in $T$ if, for every $a \in L$,

$$
\mathfrak{c}(a) \cap S=\mathrm{O} \quad \Rightarrow \quad \mathfrak{c}(a) \cap T=\mathrm{O} .
$$

We say that $S$ is codense when it is codense in $L$. Thus $S$ is codense if, for every $a \in L$,

$$
\mathfrak{c}(a) \cap S=\mathrm{O} \Rightarrow \mathfrak{c}(a)=\mathrm{O} \quad \text { (that is, } a=1) .
$$

Note. Compare this property with density, with the analogous implication concerning open sublocales. One should also keep in mind that this notion is consistent with the standard use of the term $\mathrm{co}^{-}$ dense homomorphism for the frame homomorphisms $h: L \rightarrow M$ such that $h(a)=1$ implies $a=1: S$ is codense iff the associated frame homomorphism $L \rightarrow S$ is codense iff $(a, 1) \in E_{S}$ implies $a=1$.

2.5.1. Codensity is to the operator $S^{\circ}$ as density is to the standard closure $\bar{S}$. We have

Proposition. $S$ is codense in $T$ iff $T \subseteq S^{\circ}$.

Proof. If $T \subseteq S^{\circ}$ and $S \cap \mathfrak{c}(a)=0$ then $S \subseteq \mathfrak{o}(a)$, hence $T \subseteq S^{\circ} \subseteq \mathfrak{o}(a)$, and $T \cap \mathfrak{c}(a)=0$. Thus, $S$ is codense in $T$. Conversely, if $S$ is codense in $T$ and $S \subseteq \mathfrak{o}(a)$ then $S \cap \mathfrak{c}(a)=0$, hence $T \cap \mathfrak{c}(a)=0$, and $T \subseteq \mathfrak{o}(a)$.

Then, using ??, we immediately obtain

Corollary. Let $f: L \rightarrow M$ be a localic map, $S, T \in \mathcal{S}(L)$. If $S$ is codense in $T$, then $f[S]$ is codense in $f[T]$. The converse holds whenever $f$ is injective.

\section{What happens in SPACES}

3.1. Preparing for examples. Consider an infinite set $X$ and the cofinal topology on $X$, that is, open sets are the $U_{A}=X \backslash A$ with $A$ finite, and $\emptyset$. The resulting space will be denoted by $X_{\mathrm{cf}}$.

The Heyting operation in $\Omega\left(X_{\mathrm{cf}}\right)$ is easily computed to be

$$
U_{A} \rightarrow U_{B}=U_{B \backslash A} \quad \text { and } \quad U_{A} \rightarrow \emptyset=\emptyset
$$

so that the open sublocales are

$$
\mathfrak{o}\left(U_{A}\right)=\left\{U_{B} \mid B \cap A=\emptyset\right\} \cup\{\emptyset\} \quad \text { and } \quad \mathfrak{o}(\emptyset)=0 .
$$


3.2. The spatial fitting. In analogy with ?? we define in a space $X=(X, \tau)$, for a subset $A$ of $X$, the spatial fitting

$$
A^{\circ}=\bigcap\{U \mid U \in \tau, A \subseteq U\} .
$$

Again, one has

$$
\begin{aligned}
& A \subseteq A^{\circ}, \quad A \subseteq B \Rightarrow A^{\circ} \subseteq B^{\circ}, \quad\left(A^{\circ}\right)^{\circ}=A^{\circ}, \quad \emptyset^{\circ}=\emptyset \\
& \text { and }(A \cup B)^{\circ}=A^{\circ} \cup B^{\circ} .
\end{aligned}
$$

3.2.1. Using the same symbol of $\circ$ in the superscript like in the pointfree case will, hopefully, not create confusion. One has to bear in mind, however, that the spatial fitting of a subspace (subset) in a space $X=(X, \tau)$ does not have to agree with the fitting of the associated sublocale in $\Omega(X)$. Take the space $X_{\text {cf }}$ from ??. For a subset $M \subseteq X$ one has there, trivially, $M=M^{\circ}$ but

$$
\text { for any non-empty sublocale } S \subseteq \Omega\left(X_{\text {cf }}\right), \quad S^{\circ} \text { is dense }
$$

(each non-empty open sublocale of $\Omega\left(X_{\text {cf }}\right)$ contains $\emptyset$ ) while for instance no closed sublocale $\mathfrak{c}\left(U_{A}\right)$ is dense.

3.3. The relation of subfitness and fitness to the spatial fitting is quite different from that of the fitting in the pointfree case.

First of all, due to the both-sided De Morgan formulas, unlike in the general pointfree case, the conditions

- every closed subset (resp. every subset) is an intersection of open sets,

- every open subset (resp. every subset) is an intersection of closed sets,

in spaces coincide.

We have

3.3.1. Proposition. The following statements are equivalent for a topological space $X$ :

(i) For every $M \subseteq X, M^{\circ}=M$.

(ii) Each $M \subseteq X$ is a union of closed subsets.

(iii) $X$ is $T_{1}$.

Proof. (i) $\Leftrightarrow$ (ii) by De Morgan formulas.

(ii) $\Rightarrow$ (iii): $\{x\}$ is a union of closed subsets, hence $\overline{\{x\}} \subseteq\{x\}$.

(iii) $\Rightarrow$ (ii): $M=\bigcup\{\{x\} \mid x \in M\}$. 
3.3.2. Recall that a space is said to be symmetric (see e.g. [?]) if for all $x, y \in X$

$$
x \in \overline{\{y\}} \quad \text { iff } \quad y \in \overline{\{x\}} .
$$

This is obviously equivalent with

$$
\text { for every open } U \text { and } x \in U, \quad \overline{\{x\}} \subseteq U .
$$

(Indeed, if (sym) holds, $x \in U$ and $y \in \overline{\{x\}}$ then $x \in \overline{\{y\}}$ and, since $x \in U$ open, $y \in U$. On the other hand, if (sym') holds and $x \in \overline{\{y\}}$, and if $y \in U$, then $x \in \overline{\{y\}} \subseteq U$ and hence $y \in \overline{\{x\}}$.)

3.3.3. Proposition. The following statements are equivalent for a topological space $X$ :

(i) For every closed $M \subseteq X, M^{\circ}=M$.

(ii) Each open $M \subseteq X$ is a union of closed subsets.

(iii) $X$ is symmetric.

Proof. Again, (i) $\Leftrightarrow$ (ii) by De Morgan formulas. Now we will use (sym'). (ii) $\Rightarrow$ (iii): If $x \in U$ with $U$ open then $x \in F \subseteq U$ for some closed $F$ and hence $\overline{\{x\}} \subseteq F \subseteq U$.

(iii) $\Rightarrow$ (ii): $U=\bigcup\{\overline{\{x\}} \mid x \in U\}$.

3.3.4. Note the contrast with the pointfree case: the open vs. closed conditions are here equivalent, while, on the other hand, $M^{\circ}=M$ for all $M$ and for closed $M$ are here not equivalent.

3.4. The following is a well known result $([?, ?])$. We will present a proof, because it is very short and because we wish to stress the equivalence of (1) and (2) which will contrast with ?? below.

Proposition. The following statements about a space $X$ are equivalent:

(i) $\Omega(X)$ is subfit, that is, if $A \nsubseteq B$ for open $A, B$, then there is an open $C$ such that $A \cup C=X \neq B \cup C$.

(ii) The condition (i) holds for the generic $B=X \backslash \overline{\{x\}}$ that is, for open $A$ and $x \in A$ there is an open $C$ such that $A \cup C=X \neq$ $(X \backslash \overline{\{x\}}) \cup C$.

(iii) For every open $U$ and $x \in U$ there is a $y \in \overline{\{x\}}$ such that $\overline{\{y\}} \subseteq U$.

Proof. (i) $\Rightarrow$ (ii) is trivial.

(ii) $\Rightarrow$ (iii): For an open $U$ and $x \in U$ choose an open $C$ with $U \cup C=$ $X \neq(X \backslash \overline{\{x\}}) \cup C$. Pick a $y \notin(X \backslash \overline{\{x\}}) \cup C$, that is, $y \in \overline{\{x\}}$ and $y \notin C$. Then $\overline{\{y\}} \cap C=\emptyset$ and, finally, $\overline{\{y\}} \subseteq U$.

(iii) $\Rightarrow($ i): Let $A \nsubseteq B$. Choose an $x \in A \backslash B$ and a $y \in \overline{\{x\}}$ such that $\overline{\{y\}} \subseteq A$. Set $C=X \backslash \overline{\{y\}}$. Then $A \cup C=X$ and $y \notin B \cup C$. 
Note. Compare the statement in (iii) with (sim'). Symmetry is a stronger property, not only formally so (for an example see [?]).

3.5. Fitness. In spite of the formal similarity of ?? and the statements in ??, fitness is in fact a much stronger condition than the conditions concerning $M^{\circ}=M$ for spaces (while, as shown in ??, subfitness is in fact weaker than the analogous spatial conditions). For a full characterization of fitness in spaces see [?]. Here, let us just show that even a weaker condition is close to regularity.

Recall that in Heyting terms we have $U^{*}=U \rightarrow \emptyset$. The fitness formula

$$
A \nsubseteq B \quad \Rightarrow \quad \exists C, A \vee C=X \text { and } C \rightarrow B \neq B
$$

implies a weaker prefitness [?]

$$
A \neq \emptyset \quad \Rightarrow \quad \exists D \prec A, D \neq \emptyset
$$

(indeed, in the formula above take $B=\emptyset$ and $D=C^{*}=C \rightarrow \emptyset$ ).

Now prefitness implies "regularity up to density". That is, we have

Proposition. If $\Omega(X)$ is prefit then, for every open $A$,

$$
A \subseteq \overline{\bigcup\{B \mid B \prec A\}}
$$

Proof. If $U \cap A \neq \emptyset$ choose $\emptyset \neq D \prec U \cap A$. Then $D \subseteq \bigcup\{B \mid B \subseteq$ $A\}$.

In Proposition ?? we saw that the subfitness condition could be reduced to the generic primes $X \backslash \overline{\{x\}}$ for the $B$ 's. For fitness, the situation is quite different.

3.5.1. Proposition. We have the implication

$A \nsubseteq X \backslash \overline{\{x\}} \Rightarrow \exists C$ open, $C \cup A=X$ and $C \rightarrow(X \backslash \overline{\{x\}}) \neq X \backslash \overline{\{x\}}$, for every open $A$ in $X$, iff $X$ is symmetric.

Proof. I. Explicitly, the implication says that

$x \in A \Rightarrow \exists C$ open, $C \cup A=X$ and $\operatorname{int}((X \backslash C) \cup(X \backslash \overline{\{x\}})) \supset X \backslash \overline{\{x\}}$.

Thus, there is a $y \notin X \backslash \overline{\{x\}}$, that is, $y \in \overline{\{x\}}$, and an open $U$ with $y \in U \subseteq(X \backslash C) \cup(X \backslash \overline{\{x\}})$. Since $y \in U$ open and $y \in \overline{\{x\}}, x \in U$, and hence $x \in X \backslash C$, which is closed, and finally $\overline{\{x\}} \subseteq X \backslash C \subseteq A$, the last inclusion because $C \cup A=X$.

II. Conversely, if $X$ is symmetric and $x \in A$, then $\overline{\{x\}} \subseteq A$ and we have $(X \backslash \overline{\{x\}}) \cup A=X$. Set $C=X \backslash \overline{\{x\}}$. Then

$$
C \rightarrow(X \backslash \overline{\{x\}})=C \rightarrow C=X \neq X \backslash \overline{\{x\}} \text {. }
$$


3.6. Note. In the conditions similar to fitness, very small variations can cause big differences. One may think of the relaxation to the generic elements as in ?? as fairly radical (even if such a change did not cause anything in the subfit case), but consider the following implication.

$$
a \nless \leq b \neq 0 \Rightarrow \exists c, c \vee a=1 \text { and } c \rightarrow b \neq b .
$$

Here the fitness is relaxed only by assuming the $b$ non-zero. But it is satisfied by $\Omega\left(X_{\mathrm{cf}}\right)$ which is really very far from being fit (for instance, in this frame there is no non-trivial occurrence of $x \prec y$ ). Note that, furthermore, in $\Omega\left(X_{\mathrm{cf}}\right)$ the bottom can be obtained as a meet of already two non-zero elements so that the anomaly is not caused by a special status of the bottom (in particular, this frame is subfit).

It might be of interest to discuss (fit-0), perhaps also in combination with the weak subfitness ([?], puny in [?])

$$
a \neq 0 \quad \Rightarrow \quad \exists c \neq 1, a \vee c=1
$$

to dismiss the trivial cases of the frame with immediate successor of 0 .

\section{Complete And Weakly COMplete sublocales}

4.1. A (frame) congruence is said to be complete if it is closed under arbitrary joins and arbitrary meets. For instance, each $\Delta_{a}$ (recall ??) is complete.

4.1.1. Proposition. A congruence $E \subseteq L \times L$ is complete iff each congruence class Ea contains a least element.

Proof. The implication ' $\Rightarrow$ ' is obvious.

Now consider the quotient map $q: L \rightarrow L / E$ as in ??. From the saturation formula $x E y \Rightarrow(x \leq s \equiv y \leq s)$ we immediately see that $q(a)$ is the maximum element of the equivalence class $E a$. For $x \in L / E=q[L]$ define $\phi(x)$ as the minimum element of $E x$. Then, as $\phi(x) E x$, we have

$$
q(\phi(x))=x .
$$

We claim that $\phi$ is monotone: indeed, if $x \leq y$ for $x, y \in q[L]$, we have $(\phi(x) \wedge \phi(y)) E(x \wedge y)=x E \phi(x)$ and hence $\phi(x) \wedge \phi(y)=\phi(x)$, by the minimality of $\phi(x)$. Now, by (??) and the obvious inequality

$\phi(q(x)) \leq x$, we infer that $\phi$ is a left adjoint of $q$, hence $q$ preserves all meets, and $E$ is complete. 
4.2. For a sublocale $S$, and for an element $a \in L$, set

$$
u_{a S}=\bigwedge\{x \mid \mathfrak{o}(x) \cap S=\mathfrak{o}(a) \cap S\}=\bigwedge\{x \mid \mathfrak{o}(a) \cap S \subseteq \mathfrak{o}(x)\}
$$

(the second equality holds since $\mathfrak{o}(a) \cap S \subseteq \mathfrak{o}(x)$ implies $\mathfrak{o}(a) \cap S=$ $\mathfrak{o}(x \wedge a) \cap S)$. Note that (recall ??)

$$
\{x \mid \mathfrak{o}(x) \cap S=\mathfrak{o}(a) \cap S\}=E_{S} a \quad \text { and hence } \quad u_{a S}=\bigwedge E_{S} a .
$$

4.2.1. Lemma. For any $S$ and $a \in L$,

$$
\mathfrak{o}\left(u_{a S}\right) \subseteq\left((\mathfrak{o}(a) \cap S)^{\circ}\right)^{\# \#} \subseteq(\mathfrak{o}(a) \cap S)^{\circ} .
$$

Proof. Obviously $\uparrow u_{a S} \supseteq \bigvee\{\uparrow x \mid \mathfrak{o}(a) \cap S \subseteq \mathfrak{o}(x)\}$ in $L$ and hence

$$
\begin{aligned}
\uparrow u_{a S} & \supseteq \bigvee\left\{\mathfrak{o}(x)^{\#} \mid \mathfrak{o}(a) \cap S \subseteq \mathfrak{o}(x)\right\}= \\
& =(\bigcap\{\mathfrak{o}(x) \mid \mathfrak{o}(a) \cap S \subseteq \mathfrak{o}(x)\})^{\#}=\left((\mathfrak{o}(a) \cap S)^{\circ}\right)^{\#}
\end{aligned}
$$

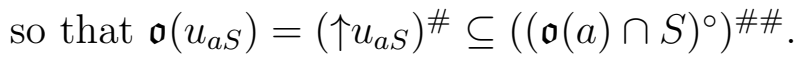

4.2.2. Lemma. If $E_{S} a$ has a least element then $(\mathfrak{o}(a) \cap S)^{\circ} \subseteq \mathfrak{o}\left(u_{a S}\right)$.

Proof. The least element is $u_{a S}$ and hence $\mathfrak{o}\left(u_{a S}\right) \cap S=\mathfrak{o}(a) \cap S$ so that $\mathfrak{o}(a) \cap S \subseteq \mathfrak{o}\left(u_{a S}\right)$ and, since $\mathfrak{o}\left(u_{a S}\right)$ is open, $(\mathfrak{o}(a) \cap S)^{\circ} \subseteq \mathfrak{o}\left(u_{a S}\right)$.

4.2.3. Proposition. $E_{S}$ a has a least element iff $(\mathfrak{o}(a) \cap S)^{\circ}$ is open.

Proof. Let $(\mathfrak{o}(a) \cap S)^{\circ}=U$ be open. Since $\mathfrak{o}(a) \cap S$ is codense in $U$, it is also codense in $\mathfrak{o}(a) \cap U$ and we see that $U=\mathfrak{o}(b)$ with $\mathfrak{o}(b) \subseteq \mathfrak{o}(a)$ and hence $b \leq a$. We have

$\uparrow b \cap S=\uparrow b \cap S \cap(\mathfrak{o}(a) \vee \uparrow a)=(\uparrow b \cap(\mathfrak{o}(a) \cap S)) \vee(\uparrow a \cap \uparrow b \cap S)=\uparrow a \cap S$ so that $b E_{S} a$. Now, if $c E_{S} a$ we have $\mathfrak{o}(a) \cap S=\mathfrak{o}(c) \cap S \subseteq \mathfrak{o}(c)$ and hence $\mathfrak{o}(b)=(\mathfrak{o}(a) \cap S)^{\circ} \subseteq \mathfrak{o}(c)$ and $b \leq c$. Thus, $b$ is the least element of $E_{S} a$.

On the other hand, if $E_{S} a$ has a least element, it is $u_{a S}$ and we have, by ?? and ??, $\mathfrak{o}\left(u_{a S}\right) \subseteq(\mathfrak{o}(a) \cap S)^{\circ} \subseteq \mathfrak{o}\left(u_{a S}\right)$, that is, $(\mathfrak{o}(a) \cap S)^{\circ}=$ $\mathfrak{o}\left(u_{a S}\right)$.

4.3. A sublocale $S$ is said to be complete if the congruence $E_{S}$ is complete.

A congruence $E$ (resp. a sublocale $S$ ) is said to be weakly complete if $E 1$ (resp. $E_{S} 1$ ) has a least element.

4.3.1. Theorem. A sublocale $S \subseteq L$ is complete iff for every open $U$, $(S \cap U)^{\circ}$ is open.

$S$ is weakly complete iff $S^{\circ}$ is open.

Proof. The first statement follows immediately from ?? and ??; for the second one apply ?? with $\mathfrak{o}(1)=L$. 
4.3.2. Notes. (1) It is easy to find examples of weakly complete but not complete sublocales (if we assume no separation axiom; but see ?? below). For instance, in the unit interval $[0,1]$ with the natural order, we have $\mathfrak{o}(a)=[0, a) \cup\{1\}$; if we consider

$$
S=\left\{s_{1}<s_{2}<\cdots<s_{n}<\cdots\right\}
$$

where $s_{n}$ converges to $\frac{1}{2}$, we have $E_{S} 1=\left[\frac{1}{2}, 1\right]$ with least element $\frac{1}{2}$, so that $S$ is weakly complete, while the other congruence classes $E_{S} a$ with $0<a<\frac{1}{2}$ are not. Or take the topology on $X=[0,1]$ consisting of the standard open sets not containing 0 , plus $X$. Then any subset containing 0 makes for a weakly complete sublocale, but many of these are not complete: for $S=\left[0, \frac{1}{2}\right]$ and $U=(0,1), S \cap U=\left(0, \frac{1}{2}\right]$ is closed (in $U$ and $X$ ) for the closure operator $(-)^{\circ}$ but $S \cap U$ is not open in $U$.

(2) A closer scrutiny of the proofs shows that we can be more specific, that is, in the case of weak completeness we know that the open set is $\mathfrak{o}\left(u_{1 S}\right)$, while in the case of completeness it is $\mathfrak{o}\left(u_{a S}\right)$. Hence, we have:

- a sublocale $S \subseteq L$ is weakly complete iff $S^{\circ}=\mathfrak{o}\left(u_{1 S}\right)$, and

$-S$ is complete iff for each $a \in L(S \cap \mathfrak{o}(a))^{\circ}=\mathfrak{o}\left(u_{a S}\right)$.

4.3.3. Recall that a localic map $f: L \rightarrow M$ is said to be open if the image of each open sublocale $S \subseteq L$ is open (note that for $T_{D^{-}}$-spaces this agrees with the open continuous maps — see [?, ?, ?]; $T_{D}$, an axiom weaker than $T_{1}$, appeared, first, in [?]).

Corollary. Open localic maps preserve weakly complete sublocales.

Proof. Let $f: L \rightarrow M$ be an open localic map and $S$ a weakly complete sublocale of $L$. By ??, $f[S]^{\circ} \supseteq f\left[S^{\circ}\right] \supseteq f[S]$. Since $S^{\circ}$ is open, $f\left[S^{\circ}\right]$ is also open and hence $f[S]^{\circ}=f\left[S^{\circ}\right]$.

4.3.4. By ??, $E_{S} 1=\{x \mid \mathfrak{o}(x) \cap S=S\}=\{x \mid S \subseteq \mathfrak{o}(x)\}$. Since $S \subseteq \mathfrak{o}(x)$ iff $S^{\circ} \subseteq \mathfrak{o}(x)$ we have

$$
E_{S^{\circ}} 1=E_{S} 1 .
$$

Lemma. If $S$ is weakly complete then

$$
\downarrow(S \backslash\{1\})=\downarrow\left(\mathfrak{o}_{1 S} \backslash\{1\}\right) .
$$

Proof. The equality states that $x\left(\operatorname{non} E_{S}\right) 1$ iff $x\left(\operatorname{non} E_{\mathfrak{o}\left(u_{1 S}\right)}\right) 1$, that is, $x E_{S} 1$ iff $x E_{\mathfrak{o}\left(u_{1 S}\right)} 1$. If $S$ is weakly complete then, by ??(2), $S^{\circ}=\mathfrak{o}\left(u_{1 S}\right)$. Use $(*)$.

4.4. Theorem. [Another characterization of subfitness] A frame $L$ is subfit iff each weakly complete sublocale of $L$ is open. 
Proof. $\Rightarrow$ : If $S$ is weakly complete then it is codense in an open $U \subseteq L$. Every open sublocale of a subfit $L$ is subfit (in fact, every complemented one is - see e.g. [?, 4.2.1]; but it also immediately follows from ??), and hence we can apply [?,5.3] and conclude that $S=U$.

$\Leftarrow$ : Let $S$ be codense in $L$. Since $L$ is open, $S$ is weakly complete and hence open, and an open codense $S \subseteq L$ is equal to $L$ (consider the complement of $S)$. Use [?, 5.3].

4.4.1. Corollary. Let $L$ be subfit. Then the following statements are equivalent.

(i) $S$ is weakly complete.

(ii) $S$ is complete.

(iii) $S$ is open.

\section{A FORMAL RELAXATION OF SUBFITNESS}

5.1. The "trivial top" characterization in terms of fitting. Yet another well known characterization of subfitness is as follows (Isbell, $[?])$ :

$$
\begin{aligned}
& \text { if } E 1=\{1\} \text { for a congruence } E \text { on } L \\
& \text { then } E=\operatorname{Id} L \text {, the identity relation on } L .
\end{aligned}
$$

This has a very concise reformulation (and a very easy proof at least in one direction) in terms of fitting.

5.1.1. Lemma. For any $S \in \mathcal{S}(L), S^{\circ}=L$ if and only if $E_{S} 1=\{1\}$.

Proof. $a E_{S} 1$ iff $a \leq s \in S$ implies that $s=1$, in other words, iff $\uparrow a \cap S=$ O. Thus, $E_{S} 1=\{1\}$ iff $S$ is codense.

5.1.2. Theorem. The following statements are equivalent for a frame $L$ :

(i) $L$ is subfit.

(ii) For any congruence $E$ of $L, E 1=\{1\} \Rightarrow E=\operatorname{Id} L$.

(iii) For any sublocale $S$ of $L, S^{\circ}=L \Rightarrow S=L$.

Proof. (iii) is by ?? only a reformulation of (ii).

(i) $\Rightarrow$ (iii): Let $L$ be subfit and let $S^{\circ}=L$. Since $L$ is open, $S$ is weakly complete by ??, hence open by ??, and consequently $S=S^{\circ}=L$.

(iii) $\Rightarrow$ (i): Let $a \not \leq b$. Hence $\uparrow b \nsubseteq \uparrow a$, and $\uparrow b \neq \uparrow b \cap \uparrow a$.

Set $S=\uparrow a \bigvee \bigvee\{\uparrow c \mid c \vee a=1\}$. Let $\uparrow x \cap \uparrow a=0$, that is, $\uparrow(a \vee x)=0$; hence $a \vee x=1$ and $\uparrow x \subseteq \bigvee\{\uparrow c \mid a \vee c=1\}$. Thus, $S$ is codense, and by the assumption, $S=L$. 
Hence, $\uparrow b=\uparrow b \cap S$, and since $\uparrow b \neq \uparrow b \cap \uparrow a$ we have

$$
\uparrow b \cap(\bigvee\{\uparrow c \mid a \vee c=1\}) \neq 0,
$$

and since $\uparrow b$ is complemented, we have, by ??, $\uparrow b \cap \uparrow c \neq 0$, that is, $b \vee c \neq 1$, for some $c$ such that $a \vee c=1$.

5.2. A formal relaxation. We will formally relax the condition of (sfit') by assuming the triviality for complete sublocales only; we will speak of $c$-subfitness. We will require for a congruence $E$ on $L$ just that

$$
\text { if } E 1=\{1\} \text { and } E \text { is complete, then } E=\operatorname{Id} L \text {. }
$$

By ??, this can be rewritten in localic terms as

$$
\text { for complete } S \text {, if } S^{\circ}=L \text { then } S=L \text {. }
$$

We will present two necessary and sufficient conditions for c-subfitness, one of them technical, another stating that this is precisely the borderline of the coincidence of completeness and openness (analogous to the characterization of subfitness in ??).

5.3. Theorem. A frame $L$ is c-subfit iff, for every complete $S$ and open $U$ in $\mathcal{S}(L)$,

$$
\downarrow(S \backslash\{1\})=\downarrow(U \backslash\{1\}) \quad \Rightarrow \quad S=U .
$$

Proof. $\Leftarrow$ is trivial.

$\Rightarrow$ : Let $\downarrow(S \backslash\{1\})=\downarrow(\mathfrak{o}(a) \backslash\{1\})$. We shall show that $\mathfrak{c}(a) \cap S=0$. Indeed, if $1 \neq s \in S$ and if $a \leq s$, then $a \in \downarrow(\mathfrak{o}(a) \backslash\{1\})$, hence $a \leq a \rightarrow x \neq 1$ for some $x$. But then $a \leq x$ and therefore $a \rightarrow x=1$, a contradiction.

5.4. Theorem. A frame $L$ is c-subfit iff each complete sublocale $S \subseteq L$ is open.

Proof. $\Rightarrow$ : Let $L$ be c-subfit and let $S \subseteq L$ be complete. Then it is weakly complete and hence, by ??, $\downarrow(S \backslash\{1\})=\downarrow\left(\mathfrak{o}\left(u_{1 S}\right) \backslash\{1\}\right)$. Thus, by ??, $S=\mathfrak{o}\left(u_{1 S}\right)$ is open.

$\Leftarrow$ : By ?? it suffices to prove that

$$
\downarrow(\mathfrak{o}(b) \backslash\{1\})=\downarrow(\mathfrak{o}(a) \backslash\{1\}) \quad \Rightarrow \quad b=a .
$$

Let the first equality hold and let $b \not \leq a$. Then $1 \neq b \rightarrow a \in \mathfrak{o}(b) \backslash\{1\}$. Then $b \rightarrow a \in \downarrow(\mathfrak{o}(a) \backslash\{1\})$ and $b \rightarrow a=a \rightarrow x$ for some $x$ such that $a \rightarrow x \neq 1$. Therefore, $a \wedge(b \rightarrow a) \leq x$ but, since $a \leq b \rightarrow a$, this yields $a \leq x$ and $a \rightarrow x=1$, a contradiction. 
5.5. Complete Heyting homomorphisms. By a theorem of Joyal and Tierney ([?]) a localic map $f: L \rightarrow M$ is open iff its associated frame homomorphism $f^{*}: M \rightarrow L$ is a complete Heyting homomorphism. On the other hand, it is known (see e.g. [?]) that for a subfit $M$ each complete frame homomorphism $h: M \rightarrow L$ is a complete Heyting homomorphism; that is, we obtain the preservation of the Heyting operation for free. Now we will show that this is true precisely for c-subfit frames.

5.5.1. Lemma. Let $h: M \rightarrow L$ be a frame homomorphism. Then we have, for congruences associated with sublocales,

$$
(h \times h)^{-1}\left[E_{S}\right]=E_{h_{*}[S]} .
$$

Proof. Use ??. From the adjunction $h \dashv h_{*}$ we have that $(x, y) \in$ $(h \times h)^{-1}\left[E_{S}\right]$, that is,

$$
\forall s \in S, \quad h(x) \leq s \text { iff } h(y) \leq s
$$

if and only if

$$
\forall s \in S, \quad x \leq h_{*}(s) \text { iff } y \leq h_{*}(s),
$$

that is, $(x, y) \in E_{h_{*}[S]}$.

5.5.2. Theorem. Each complete frame homomorphism $h: M \rightarrow L$ is a complete Heyting homomorphism iff $M$ is c-subfit.

Proof. $\Leftarrow$ : Let $M$ be c-subfit and let $h: M \rightarrow L$ be a complete frame homomorphism. Let $S$ be an open sublocale of $L$. Then, in particular, it is complete, and hence $E_{S}$ is complete. From the completeness of $h$ and from ?? we now immediately infer that $E_{h_{*}[S]}$ is complete. Thus, $h_{*}[S]$ is complete and, by ??, it is open. We conclude that $h$ is an open homomorphism and apply the Joyal-Tierney theorem.

$\Rightarrow$ : Let $S$ be a complete sublocale of $M$. Consider the quotient homomorphism $h: M \rightarrow S$ adjoint to the embedding $j: S \subseteq M$. Then $h$ is a complete homomorphism and hence it is a Heyting one. Thus, it is an open homomorphism and, in particular, $S=j[S]$ is an open sublocale.

5.6. Note. Restricting in (c-sfit) the condition from general sublocales to (very special) complete ones seems to be a very radical reduction. Yet, it is still an open problem whether it is not, after all, just another formula for subfitness. It has defied solution for years, even in modified contexts like that of Heyting meet-semilattices ([?]).

Any answer to this problem would be of interest. And if it should turn out that c-subfitness were strictly weaker then fitness, it would be of interest to find a first order formula akin to (sfit) in ??. 
Acknowledgements. This work was partially supported by the Centre for Mathematics of the University of Coimbra (UID/MAT/00324/ 2013 funded by the Portuguese Government through FCT/MCTES and co-funded by the European Regional Development Fund through the Partnership Agreement PT2020), and by projects P202/12/G061 (Grant Agency of the Czech Republic) and MTM2015-63608-P (Ministry of Economy and Competitiveness of Spain). The first author also acknowledges a sabbatical grant from FCT (grant SFRH/BSAB/127925 /2016).

Thanks are due to the referee for comments and suggestions that have helped improve the presentation of the paper.

\section{REFERENCES}

[1] C.E. Aull and W.J. Thron, Separation axioms between $T_{0}$ and $T_{1}$, Indag. Math. 24 (1963) 26-37.

[2] B. Banaschewski and A. Pultr, Variants of openness, Appl. Categ. Structures 2 (1994) 331-350.

[3] D. Dikranjan and E. Giuli, Closure operators I, Topology Appl. 27 (1987) 129143.

[4] C.H. Dowker and D.P. Strauss, Separation axioms for frames, Colloq. Math. Soc. Janos Bolyai 8 (1974) 223-240.

[5] C.H. Dowker and D. Strauss, $T_{1^{-}}$and $T_{2}$-axioms for frames, in: Aspects of topology, London Math. Soc. Lecture Note Ser. 93, pp. 325-335, Cambridge Univ. Press, Cambridge, 1985.

[6] T. Dube, A note on weakly pseudocompact locales, Appl. Gen. Topol. 18 (2017) 131-141.

[7] H. Herrlich, A concept of nearness, Gen. Topology Appl. 5 (1974) 191-212.

[8] H. Herrlich and A. Pultr, Nearness, subfitness and sequential regularity, Appl. Categ. Structures 8 (2000) 67-80.

[9] J.R. Isbell, Atomless parts of spaces, Math. Scand. 31 (1972) 5-32.

[10] P.T. Johnstone, Stone Spaces, Cambridge Univ. Press, Cambridge, 1982.

[11] A. Joyal and M. Tierney, An extension of the Galois theory of Grothendieck, Mem. Amer. Math. Soc., no. 309, 1984.

[12] S. Mac Lane, Categories for the Working Mathematician, Springer-Verlag, New York, 1971.

[13] J. Picado and A. Pultr, Frames and Locales: topology without points, Frontiers in Mathematics, Vol. 28, Springer, Basel, 2012.

[14] J. Picado and A. Pultr, More on subfitness and fitness, Appl. Categ. Structures 23 (2015) 323-335.

[15] J. Picado and A. Pultr, New aspects of subfitness in frames and spaces, Appl. Categ. Structures 24 (2016) 703-714.

[16] J. Picado, A. Pultr and A. Tozzi, Ideals in Heyting semilattices and open homomorphisms, Quaest. Math. 30 (2007) 391-405.

[17] T. Plewe, Quotient maps of locales, Appl. Categ. Structures 8 (2000) 17-44.

[18] H. Simmons, The lattice theoretic part of topological separation properties, Proc. Edinburgh Math. Soc. (2) 21 (1978) 41-48. 
[19] H. Simmons, Regularity, fitness, and the block structure of frames, Appl. Categ. Structures 14 (2006) 1-34.

[20] Alex Simpson, Measure, randomness and sublocales, Ann. Pure Appl. Logic 163 (2012) 1642-1659.

(M. M. Clementino and J. Picado) CMUC, Department of Mathematics, University of Coimbra, 3001-501 Coimbra, Portugal

E-mail address: mmc@mat.uc.pt, picado@mat.uc.pt

(A. Pultr) Department of Applied Mathematics and iti, MFF, Charles University, Malostranské nám. 24, 11800 Praha 1, Czech Republic

E-mail address: pultr@kam.mff.cuni.cz 


\title{
Correction to: The Other Closure and Complete Sublocales
}

\author{
Maria Manuel Clementino ${ }^{1} \cdot$ Jorge Picado $^{1} \cdot$ Aleš Pultr $^{2}$ \\ Published online: 29 June 2018 \\ (c) Springer Nature B.V. 2018
}

\section{Correction to: Appl Categor Struct https://doi.org/10.1007/s10485-018-9516-4}

In the original publication of the article, the formulation of the $c$-subfitness condition (c-sfit) in Subsection 5.2 is inaccurate, with effect in Theorem 5.3. A corrected version of 5.2 and more explanation in the proof of 5.3 is provided in this Corrigendum note.

\subsection{A Formal Relaxation}

Since an open sublocale of a subfit locale is subfit, we can replace (iii) in 5.1.2 by a formally stronger claim concerning an arbitrary open $U \subseteq L$ instead of $L$. Thus, we can characterize subfitness by

$$
\text { for any open } U \subseteq L \text { and any sublocale } S \subseteq L, \quad S^{\circ}=U \Rightarrow S=U
$$

(compare with 4.3). Now, we will formally relax this condition to

$$
\begin{gathered}
\text { for any open } U \subseteq L \text { and any complete sublocale } S \subseteq L, \\
S^{\circ}=U \Rightarrow S=U .
\end{gathered}
$$

We will present two necessary and sufficient conditions for c-subfitness, one of them technical, another stating that it is precisely the borderline of the coincidence of completeness and openness.

The original article can be found online at https://doi.org/10.1007/s10485-018-9516-4.

Jorge Picado

picado@mat.uc.pt

Maria Manuel Clementino

mmc@mat.uc.pt

Aleš Pultr

pultr@kam.mff.cuni.cz

1 CMUC, Department of Mathematics, University of Coimbra, 3001-501 Coimbra, Portugal

2 Department of Applied Mathematics and ITI, MFF, Charles University, Malostranské nám. 24,

11800 Praha 1, Czech Republic 
5.3 Theorem A frame $L$ is c-subfit iff, for every complete $S$ and open $U$ in $\mathcal{S}(L)$,

$$
\downarrow(S \backslash\{1\})=\downarrow(U \backslash\{1\}) \Rightarrow S=U .
$$

Proof $\Rightarrow$ : Since $S^{\circ}=\mathfrak{o}(a)$ is obviously the same as claiming that $S \subseteq \mathfrak{o}(a)$ together with $\downarrow(S \backslash\{1\})$ being cofinal in $\downarrow(\mathfrak{o}(a) \backslash\{1\})$, it suffices to prove that $S \subseteq \mathfrak{o}(a)$.

Let $\downarrow(S \backslash\{1\})=\downarrow(\mathfrak{o}(a) \backslash\{1\})$. We shall show that $\mathfrak{c}(a) \cap S=\mathrm{O}$. Indeed, if $1 \neq s \in S$ and $a \leq s$, then $a \in \downarrow(\mathfrak{o}(a) \backslash\{1\})$, hence $a \leq a \rightarrow x \neq 1$ for some $x$. But then $a \leq x$ and therefore $a \rightarrow x=1$, a contradiction.

$\Leftarrow$ is trivial. 